\title{
Dual Regulation of Calcium Oscillation in Astrocytes by Growth Factors and Pro-Inflammatory Cytokines via the Mitogen-Activated Protein Kinase Cascade
}

\author{
Mitsuhiro Morita, ${ }^{1}$ Chitose Higuchi, ${ }^{1}$ Takanori Moto, ${ }^{1}$ Nagisa Kozuka, ${ }^{1}$ Jinichi Susuki, ${ }^{1}$ Rurika Itofusa, ${ }^{1}$ \\ Jiro Yamashita, ${ }^{2}$ and Yoshihisa Kudo ${ }^{1}$ \\ ${ }^{1}$ Laboratory of Cellular Neurobiology, School of Life Science, Tokyo University of Pharmacy and Life Science, 192-0392 Tokyo, Japan, and ${ }^{2}$ Department of \\ Neurophysiology, University of Tokyo, Graduate School of Medicine, 113-0033 Tokyo, Japan
}

\begin{abstract}
In response to neurotransmitters, astrocytes show various types of calcium increase (transient, oscillatory, and complex), the physiological significance of which is still controversial. To explore this variability, we examined factors affecting the calcium increase pattern in cultured astrocytes and investigated the consequences of the astrocytic calcium response in slice preparations. We found that growth factors (GFs) (EGF plus basic FGF) promoted calcium oscillation in response to glutamate, ATP, or thimerosal (which directly activates the inositol-1,4,5 triphosphate receptor) and that this effect was suppressed by pro-inflammatory cytokines (interleukin- $1 \beta$ or tumor necrosis factor- $\alpha$ ), lipopolysaccharide, or a MEK (mitogen-activated protein kinase kinase) inhibitor, suggesting dual regulation of calcium oscillation in astrocytes by factors affecting brain function and pathology via the mitogen-activated protein kinase (MAPK) cascade. The calcium oscillation was accompanied by enlargement of the calcium store, cell proliferation, and the development of a hypertrophic morphology. The cytokines suppressed GF-induced MAPK-dependent immediate early gene promoter activation, but not phosphorylation of extracellular signal-regulated kinase (ERK), showing that they affected gene regulation by acting on the MAPK cascade downstream of ERK. In slice preparations, a metabotropic glutamate receptor agonist converted the spontaneous neuronal calcium increase, attributable to synaptic transmission, to an oscillatory response similar to that seen in astrocytes in culture, indicating that the calcium response in astrocytes acted as a feedback mechanism on the activity of neighboring neurons. This is the first evidence for a dual regulation of calcium oscillation by physiological factors and for the control of calcium dynamics actually being used in physiological processes.
\end{abstract}

Key words: astrocyte; calcium oscillation; epidermal growth factor; basic fibroblast growth factor; lipopolysaccharide; interleukin-1 $\beta$; tumor necrosis factor- $\alpha$; mitogen-activated protein kinase; metabotropic glutamate receptor; inositol-1,4,5 triphosphate

\section{Introduction}

Calcium imaging and the identification of receptor genes have provided useful information about the astrocytic calcium response to neurotransmitters (Verkhratsky et al., 1998) and have suggested a dynamic role for astrocytes in brain function (Haydon, 2001). Astrocytes, which form the largest population of nonexcitable cells in the mammalian CNS, adhere closely to neurons and blood vessels and fill the space between them (Zonta et al., 2003). This anatomical property is advantageous not only in the regulation of CNS metabolism but also for the interaction of

Received June 12, 2003; revised Sept. 11, 2003; accepted Oct. 2, 2003.

This work was supported by Grant-in Aid 10214204 for Scientific Research on Priority Areas (B) on "Regulation of Neural Transduction by Glial Cells" and Grant-in Aid 15082101 for Scientific Research on Priority Areas on "Elucidation of Glia-Neuron Network-Mediated Information Processing Systems," both from the Ministry of Education, Science and Culture in Japan. We thank Dr. Hiroyoshi Miyakawa and Dr. Kazuhiro Ikenaka for critical reading of this manuscript. We thank Ryuichi Nakajima for technical assistance. We also thank Hiromi Yanaka and Keiko Suzuki for excellent secretarial assistance.

Correspondence should be addressed to Mitsuhiro Morita, Laboratory of Cellular Neurobiology, School of Life Science, Tokyo University of Pharmacy and Life Science, 1432-1, Horinouchi, Hachioji, 192-0392 Tokyo, Japan. E-mail:moritam@|s.toyaku.ac.jp.

Copyright $\odot 2003$ Society for Neuroscience ～0270-6474/03/2310944-09\$15.00/0 astrocytes with neurotransmitters released from nerve terminals. During neuronal activity, astrocytes respond by an increase in intracellular calcium levels (Pasti et al., 1997), but many aspects of this response have not been well studied, for instance, the degree of neuronal activity required and the consequences for brain function.

In this study, we cultured astrocytes in serum-free defined medium and examined the effects of growth factors (GFs) and cytokines on calcium dynamics. Although astrocytes have been studied by calcium imaging for $>10$ years, their reported pharmacological properties and response pattern vary (transient or oscillatory), depending on the species, brain region, and age of the animal (McCarthy and Salm, 1991; Muller et al., 1997; Cai et al., 2000), and this complexity makes it difficult to determine the physiological role of these phenomena. We propose that this variability could reflect an important function of astrocytes, namely that they can respond to factors, such as ions, neurotransmitters, bioactive lipid metabolites, GFs, cytokines, and adhesion molecules, in the CNS environment and can alter the environment by changing their morphology and metabolism, including the expression of enzymes and other factors (Rostworowski et al., 1997; 
Stachowiak et al., 1997; Krushel et al., 1998; Verkhratsky et al., 1998; Xian and Zhou, 1999; Bezzi et al., 2001). These responses are important in maintaining CNS homeostasis but would cause significant variation in the results of studies on cultured astrocytes. Small differences in culture conditions, such as cell density, serum, and the presence of other cell populations, affect astrocyte metabolism by the presence of soluble factors or by cell adhesion, leading to further changes in the medium. Because of this synergistic effect, initial small differences can produce markedly different results. To avoid these issues and to examine the real properties of astrocytes, it is necessary to culture them in well defined conditions, for which serum-free defined medium is ideal. The use of defined medium allows the effect of the environment on the physiological properties of astrocytes to be systematically studied and should provide important new information on astrocyte function. Calcium oscillation is reported to generate repetitive glutamate release from astrocytes that then affects surrounding neurons (Pasti et al., 1997, 2001), suggesting a feedback mechanism from the astrocyte to the neuronal network. We therefore studied calcium oscillation in astrocytes cultured in defined medium and compared these responses with those in tissue slice preparations to determine whether our culture system results could be applied to in vivo studies.

\section{Materials and Methods}

Cell culture and calcium imaging. Astrocytes were isolated from the cerebral cortex of postnatal day 1 Wistar rats, using a modification of the method described by Levison and McCarthy (1991). Briefly, brain cells were prepared from the cortices of $10-15$ brains by trypsinization, trituration, and filtration, and seeded at $1.3 \times 10^{4}$ cells $/ \mathrm{cm}^{2}$ in $75 \mathrm{~cm}^{2}$ plastic flasks (Sumitomo Bakelite, Tokyo, Japan). After culture for $12 \mathrm{~d}$ at $37^{\circ} \mathrm{C}$ in $5 \% \mathrm{CO}_{2}$ humidified air in basal Eagle's medium containing $10 \%$ fetal calf serum (FCS) (Equitech-Bio, Ingram, TX) with a medium change every $3 \mathrm{~d}$, the resulting mixed glia culture was shaken at $260 \mathrm{rpm}$ for $18 \mathrm{hr}$ at $37^{\circ} \mathrm{C}$ and then rinsed with medium to remove nonastrocytic cells. The adherent cells were subcultured by trypsinization and seeded at a density of $3 \times 10^{4}$ cells $/ \mathrm{cm}^{2}$ in DMEM (Asahi Technoglass, Funabashi, Japan) containing 25 mM HEPES, pH 7.4, and 10\% FCS on either polyD-lysine-coated coverslips (for calcium imaging or immunocytochemistry) or plastic plates (Asahi Technoglass) (for Western blotting or reporter gene assays). After $48 \mathrm{hr}$, the medium was changed to that described in each experiment, and culture continued for an additional $48-96 \mathrm{hr}$ before assay. The astrocyte-defined medium (ADM), a modified version of that reported by Miller et al. (1995), consisted of DMEM containing $25 \mathrm{~mm}$ HEPES, pH 7.4, $1 \mathrm{~mm}$ pyruvate, $2 \mathrm{~mm}$ glutamine, 50 $\mathrm{mg} / \mathrm{ml}$ human apotransferrin (Invitrogen, Gaithersburg, MD), $10 \mathrm{ng} / \mathrm{ml}$ D-biotin, $5.2 \mathrm{ng} / \mathrm{ml}$ sodium selenite, $1.5 \mathrm{mg} / \mathrm{ml}$ bovine fibronectin (Invitrogen), $0.5 \mathrm{mg} / \mathrm{ml}$ heparin sulfate, $5 \mathrm{mg} / \mathrm{ml}$ insulin (Invitrogen), 10 $\mathrm{ng} / \mathrm{ml}$ epidermal growth factor (EGF) (Invitrogen), and $5 \mathrm{ng} / \mathrm{ml}$ basic fibroblast growth factor (bFGF) (Invitrogen); GF-free ADM had the same composition, but lacked the EGF and bFGF. Rat interleukin- $1 \beta$ (IL-1 $\beta)$ and rat tumor necrosis factor- $\alpha(\mathrm{TNF} \alpha)$ were obtained from Peprotech (London, UK). For calcium imaging, the cells were washed three times and then loaded for $45 \mathrm{~min}$ at $37^{\circ} \mathrm{C}$ with $7.5 \mu \mathrm{M}$ fura- $2 \mathrm{AM}$ (Dojin, Kumamoto, Japan) in basal salt saline (BSS) consisting of (in mM): $129 \mathrm{NaCl}, 4 \mathrm{KCl}, 1 \mathrm{MgCl}_{2}, 2 \mathrm{CaCl}_{2}, 4.2$ glucose, and $10 \mathrm{HEPES}$, the $\mathrm{pH}$ being adjusted to 7.4 with $\mathrm{NaOH}$. In all steps after loading, $100 \mu \mathrm{M}$ sulfinpyrazone was added to the BSS. After washing and incubation at room temperature for $20 \mathrm{~min}$, the coverslips were mounted in a chamber (RC-26; Warner Instruments, Hamden, CT) and perfused at a rate of 1.5 $\mathrm{ml} / \mathrm{min}$. Calcium imaging was performed using an IX70 inverted microscope, a UApo/340 40X/1.15w objective, and an OSP-EXA filter exchanger (all from Olympus, Tokyo, Japan) equipped with a C6790 CCD camera (Hamamatsu Photonics, Hamamatsu, Japan). Fluorescence images were acquired and ratio images (R340/380) calculated using AQUACOSMOS software (Hamamatsu Photonics). For quantitative analysis of calcium oscillation, the nuclei were visualized by staining using acridine orange and UV illumination, a newly developed method (Morita et al., 2003). In each imaging experiment, 20-40 cells were identified; each histogram shows the results from six imaging experiments from two series of cultures prepared from two different preparations of cells.

Immunological detection. Immunocytochemical staining for glial fibrillary acidic protein (GFAP) was performed using a rabbit polyclonal anti-GFAP antibody (5 $\mu \mathrm{g} / \mathrm{ml}$; Dako, Hamburg, Germany) and rhodamine-conjugated goat second antibody $(5 \mu \mathrm{g} / \mathrm{ml}$; Chemicon, Temecula, CA). For counterstaining of the nucleus, Hoechst $33258(1 \mu \mathrm{M})$ was added to the second antibody solution. To assess metabotropic glutamate receptor-5 (mGluR5) expression, Western blotting was performed using rabbit polyclonal anti-mGluR5 antibody ( $2 \mu \mathrm{g} / \mathrm{ml}$; Upstate Biotechnology, Lake Placid, NY) or mouse monoclonal anti-actin antibody (1:1000 dilution; Chemicon). To assess extracellular signalregulated kinase (ERK) phosphorylation, cells on six-well plates were cultured in GF-free ADM for $48 \mathrm{hr}$ and pretreated either overnight with cytokines or lipopolysaccharide (LPS) or for $5 \mathrm{~min}$ with the mitogenactivated protein (MAP) kinase kinase (MEK) inhibitor; then GFs were added for $5 \mathrm{~min}$, and Western blotting was performed using mouse monoclonal antibody against phosphorylated ERK ( $2 \mu \mathrm{g} / \mathrm{ml}$; Sigma, St. Louis, MO) or rabbit polyclonal anti-ERK antibody $(0.6 \mu \mathrm{g} / \mathrm{ml}$; Sigma $)$. Horseradish peroxidase-conjugated secondary antibody from Amersham Biosciences (Buckinghamshire, UK) was used to detect all bound primary antibodies.

Reporter gene assay. The promoter region of the rat early growth response gene-1 (egr-1) gene $(-525$ to +117$)$ (Changelian et al., 1989) was obtained by PCR and subcloned into pGL3-Basic (Promega, Madison, WI). This reporter gene vector was transfected, using TransFast (Promega), into astrocytes that had been grown for $48 \mathrm{hr}$ in DMEM containing 25 mM HEPES, pH 7.4, and 1\% FCS. After $24 \mathrm{hr}$, the medium was changed to GF-free ADM, and then, after $48 \mathrm{hr}$ culture, with or without pretreatment, as described for the Western blot experiments above, GFs were added for $6 \mathrm{hr}$, and luciferase activity was assayed using PicaGene (Nippon Gene, Tokyo, Japan).

Slice culture and calcium imaging. Slice cultures were prepared from the hippocampus of postnatal day 7 Wistar rats, as described previously (Hirasawa et al., 2000), and cultured for 7-14 d before calcium imaging. BSS containing $0.1 \mathrm{~mm}$ ascorbic acid and $0.5 \mathrm{~mm}$ inositol was used throughout, and sulfinpyrazone was included as described for the cell culture experiments. The cells were incubated with $50 \mu \mathrm{M}$ MK801 for 30 min before and during loading for $1 \mathrm{hr}$ at $37^{\circ} \mathrm{C}$ with fluo-4 AM (Molecular Probes, Eugene, OR) in BSS containing 0.005\% Cremophore. After three washes, the slices were incubated for $30 \mathrm{~min}$ at room temperature in medium without MK801 and then were transferred for 5 min to BSS containing $100 \mathrm{~mm}$ mannitol, which suppresses swelling during pharmacological stimulation. Calcium imaging was performed using an E600FN upright microscope and a Fluor $40 \times / 0.8 \mathrm{w}$ objective (both from Nikon, Tokyo, Japan) equipped with a CSU-10 laser confocal scanning unit (Yokokawa, Tokyo, Japan), a 532R-BS-A04 argon laser (Melles Griot, Irvine, CA), and a C6790 CCD camera (Hamamatsu). Fluorescence images were acquired using AQUACOSMOS software (Hamamatsu), and the fluorescence ratio $(F / F o)$ was calculated from the average intensity of the indicated areas.

\section{Results}

\section{Growth factor-induced calcium oscillation}

As in a previous report (Jensen and Chiu, 1990), astrocytes cultured in medium containing 10\% FCS, a commonly used additive, were found to consist of a mixture of two populations, the proportions of which varied between cultures. One of these showed a transient response, and the other an oscillatory response, to glutamate $(30 \mu \mathrm{M})$ or ATP $(100 \mu \mathrm{M})$ (Fig. $1 A$, top panels); the percentage of responding cells showing oscillatory responses to glutamate or ATP, respectively, was 33.3\% $(n=42)$ and $18.9 \%(n=58)$. In contrast, after culture for $48-96 \mathrm{hr}$ in serum-free defined medium containing EGF and bFGF (ADM), almost all of the responding cells showed calcium oscillation 
(center panels). Typical imaging data for the calcium oscillation in response to glutamate are shown in the supplementary data (movie 1; available at www.jneurosci.org). Furthermore, these cells showed a similar oscillatory response to thimerosal $(10 \mu \mathrm{M})$, which affects the redox state of the inositol-1,4,5 triphosphate $\left(\mathrm{IP}_{3}\right)$ receptor and induces calcium release (Swann, 1991). In contrast, cells in GF-free ADM gave a transient response to all three stimuli (bottom panels). The percentage of responding cells showing oscillatory responses to glutamate, ATP, or thimerosal, respectively, was $10.3 \%(n=156), 8.3 \%$ $(n=60)$, and $3.6 \%(n=56)$ in GF-free $\mathrm{ADM}$ and $75.0 \%(n=212), 74 \%(n=85)$, and $80.0 \%(n=128)$ in ADM.

Although it has been reported previously that this same set of GFs increases astrocyte mGluR5 expression, enhances phosphoinositide hydrolysis and the calcium response, and converts the calcium response to oscillatory (Miller et al., 1995; Nakahara et al., 1997), Western blotting showed no significant increase in mGluR5 protein levels in the presence of GFs over the same time course (Fig. $1 B$ ). These results show that the mixed calcium response seen in serum-containing medium could be converted to an entirely transient or entirely oscillatory response in serum-free medium depending on the absence or presence, respectively, of GFs, and that this conversion was mediated by changes in some calciumcontrolling mechanism. Because the effects of defined medium required $>48 \mathrm{hr}$ to become apparent (data not shown), we hypothesized that regulation of gene expression was involved and that the candidate genes would be those coding for proteins regulating intracellular calcium dynamics, such as calcium channels, pumps, exchangers, and buffer proteins.

\section{Inhibition by cytokines or a MEK inhibitor}

GF production in the CNS changes during development, under different pathological conditions, and during functions such as memory formation and has been shown to affect astrocyte proliferation and their differentiation to reactive astrocytes (Stachowiak et al., 1997; Xian and Zhou, 1999). Production of proinflammatory cytokines, such as IL1 $\beta$ and $\operatorname{TNF} \alpha$, also changes with pathology and stress and is known to affect astrocyte proliferation, morphology, and metabolism (Rostworowski et al., 1997; Murray and Lynch, 1998; Herx and Yong, 2001). These two groups of factors (GFs and pro-inflammatory cytokines) competitively regulate the production of $S 100 \beta$ or growth inhibitory factor by astrocytes (Hinkle et al., 1998; Uchida, 1999) and are produced with different time courses in brain injury, cytokine levels increasing within several hours after insult (Rostworowski et al., 1997), whereas GF levels reach a maximum only after 1 week, when the scar of astrocytes becomes mature (Iseki et al., 2002). On the basis of these results, we hypothesized that these soluble factors may have opposing effects and examined the effect of cytokines on the GF-induced calcium oscillation in astrocytes. We also examined the effects of LPS, which is known to activate a cellsignaling cascade similar to that activated by pro-inflammatory cy- tokines (Raetz and Whitfield, 2002), and those of a MEK inhibitor, U0126, which attenuates the MAPK cascade, one of the main pathways activated by GFs (Favata et al., 1998).

Figure $2 \mathrm{~A}$ shows calcium responses of three representative cells cultured in ADM containing a pro-inflammatory cytokine (IL1 $\beta$ or TNF $\alpha$ ), LPS, or a MEK inhibitor, all of which suppressed the calcium oscillation induced by the GFs. To analyze these results quantitatively, individual cells were identified by nuclear staining with acridine orange after calcium imaging with fura-2 AM, and their calcium responses to $30 \mu \mathrm{M}$ glutamate were measured and plotted as frequency and amplitude histograms (Figs. $2 B, C$ ). The number of peaks seen during 2 min stimulation with glutamate was $4.12 \pm 0.20$ (mean \pm SEM; $n=228$ ) and $1.04 \pm 0.05(n=178)$ in ADM and GF-free ADM, respectively, indicating that the GFs caused a marked change in the pattern. Although the various agents tested gave different effects, giving frequencies between 0.90 and 2.04 peaks per $2 \mathrm{~min}$ in all cases, fewer peaks were seen than in ADM alone. The amplitude of the calcium response was also suppressed in parallel with the calcium oscillation. Because the percentage of cells showing no response increased from $7.0 \%$ in $\mathrm{ADM}$ and $9.8 \%$ in GF-free $\mathrm{ADM}$ to $24.9 \%$ in ADM plus LPS, $35.5 \%$ in ADM plus IL1 $\beta, 56.7 \%$ in $\mathrm{ADM}$ plus $\mathrm{TNF} \alpha$, and $47.4 \%$ in ADM plus U0126, the calcium responses were recalculated using only the responding cells, with similar results (shown in parentheses).

\section{Enlargement of calcium stores}

In astrocytes, glutamate presumably acts by inducing $\mathrm{IP}_{3}$ induced calcium release via mGluRs, because the calcium response was not induced by either kainic acid or NMDA, both of which act on ion channel-type glutamate receptors, but was induced by the group I mGluR agonist ( $S$ )-3,5-dihydroxyphenylglycine or the more selective mGluR5 agonist $(R S)$-2-chloro-5- 

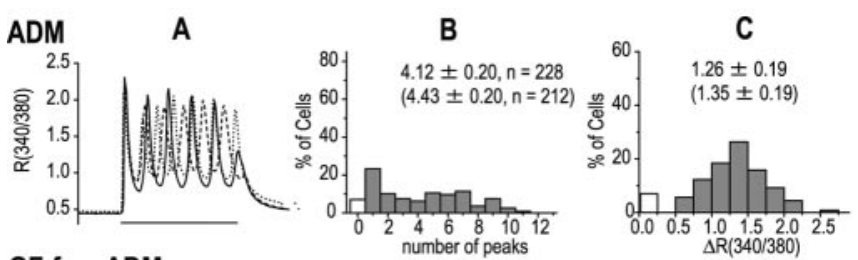

\section{GF-free ADM}
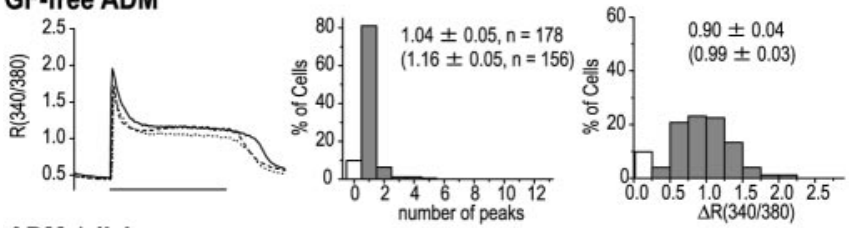

ADM+IL1
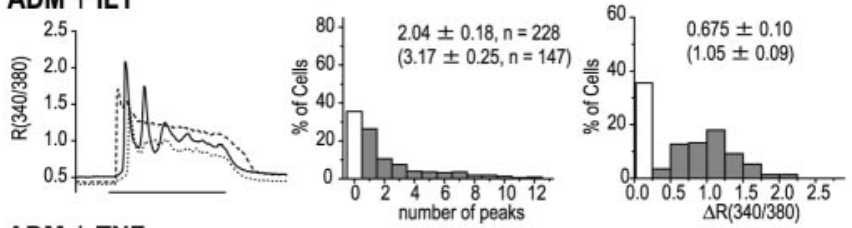

$\mathrm{ADM}+\mathrm{TNF}$
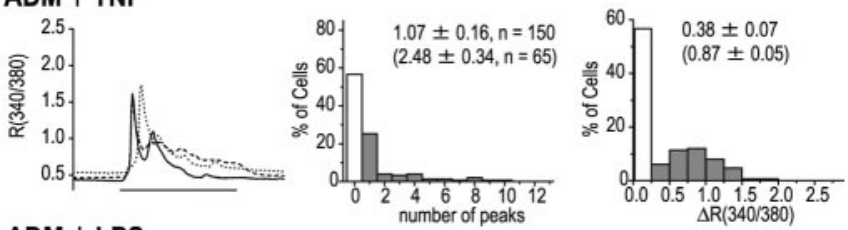

\section{$\mathrm{ADM}+\mathrm{LPS}$}
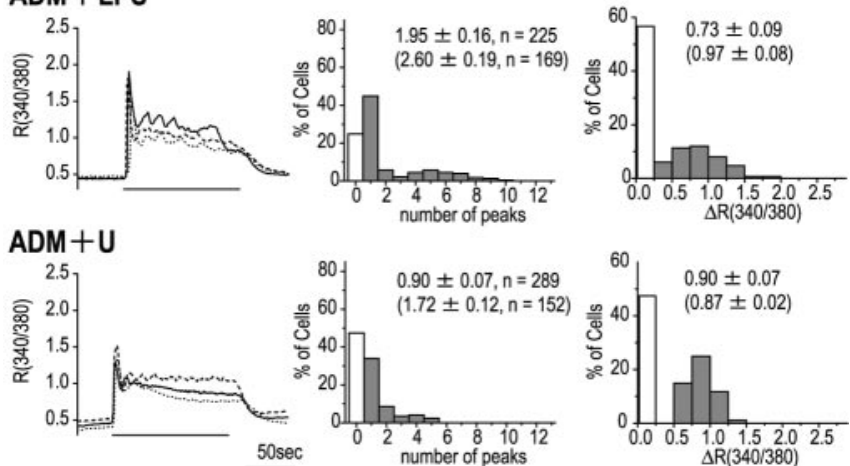

Figure 2. Factors affecting the astrocytic calcium response. Calcium responses of astrocytes to $30 \mu \mathrm{m}$ glutamate after $48-72 \mathrm{hr}$ culture in various media. The effects of two proinflammatory cytokines, IL-1 $\beta$ (IL $1 ; 5 \mathrm{ng} / \mathrm{ml})$ and TNF $\alpha$ (TNF; $20 \mathrm{ng} / \mathrm{ml}), \mathrm{LPS}(0.01 \mu \mathrm{g} / \mathrm{ml})$, or the MEK inhibitor U0126 $(\mathrm{U} ; 20 \mu \mathrm{M})$ were examined in cells cultured in ADM. A, Representative calcium responses from three cells under the different experimental conditions. $B$, Histograms showing the frequency of calcium oscillation. $C$, Histograms showing the maximum amplitude of the calcium response. The white columns indicate cells showing no calcium response. The higher numerical values in each panel are the mean \pm SEM and number of cells $(n)$ for all cells (responders plus nonresponders), whereas the lower values (shown in parentheses) are those for responding cells.

hydroxyphenylglycine (Conn and Pin, 1997) (data not shown). Because direct activation of the $\mathrm{IP}_{3}$ receptor with thimerosal was sufficient to induce an oscillatory calcium response, the regulatory mechanisms of intracellular calcium dynamics were assumed to be the main target of factors affecting calcium oscillation, and we therefore investigated changes in the calcium store. To compare the sizes of the calcium store involved, the cells were treated with ionomycin in the absence of extracellular calcium, and the amount of released calcium was measured; this treatment abolished the glutamate-induced calcium release (Fig. $3 A$, left), showing that it depleted the store required for calcium oscillation. In the absence of ionomycin treatment, astrocytes retained the ability to release calcium even after $6 \mathrm{~min}$ in the absence of
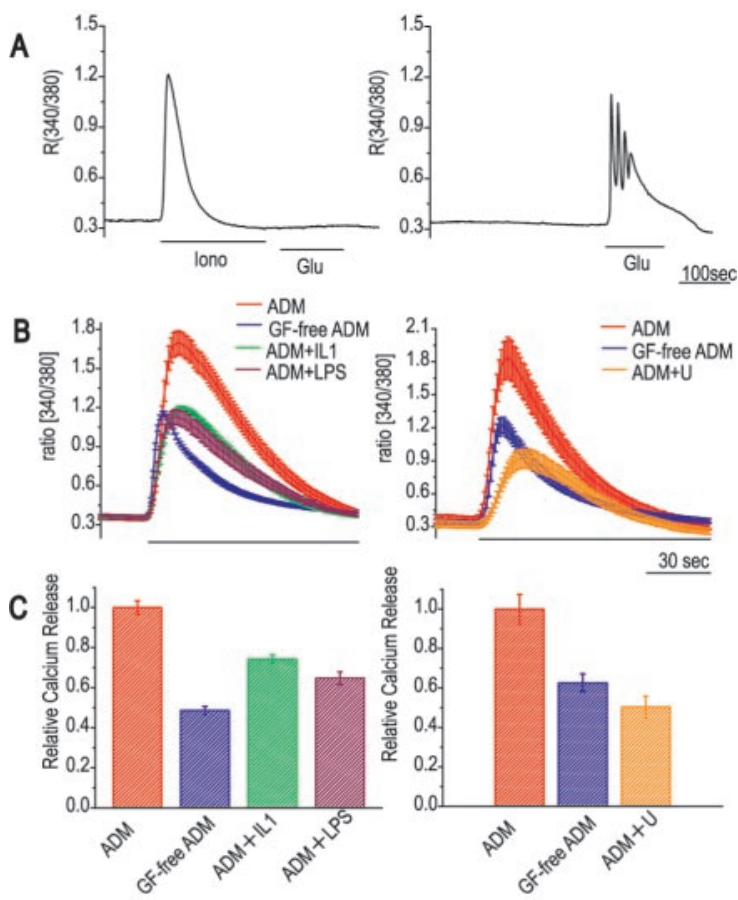

Figure 3. The size of the calcium store is altered by factors affecting calcium oscillation. $A$, Procedure for assessing the size of the calcium store. Astrocytes cultured in ADM were transferred to calcium-free medium at the start of the trace and then treated either with ionomycin (Iono; $2.5 \mu \mathrm{m}$ ) followed by glutamate (Glu; $30 \mu \mathrm{m}$ ) (left panel) or with glutamate alone (right panel). Ionomycin causes calcium release, which represents the size of the calcium store, and abolishes the calcium response to glutamate. $B$, lonomycin-induced calcium release of astrocytes cultured under different conditions; ionomycin treatment is indicated by the bar underneath the traces. Each trace is the mean \pm SEM for three cells in a single imaging experiment. The effects of cytokines (left panel) and the MEK inhibitor (right panel) were compared in the same series of cultures from a single batch of cells. C, Quantitative comparison of calcium release. The ionomycin-induced calcium release from cells cultured in different media is expressed relative to that of cells cultured in ADM. The values are the mean \pm SEM for 21 cells from three independent imaging experiments using the same series of cultures as in $B$.

extracellular calcium (Fig. $3 A$, right). Figure $3 B$ shows calcium release in astrocytes under different culture conditions. When we used this method to compare the size of the stores under each of these conditions, the calcium release in the presence of GFs was approximately twice that seen in their absence (Fig. $3 C$ ) and was decreased to an intermediate level by the presence of proinflammatory cytokines, LPS, or the MEK inhibitor, indicating that the enlargement of the calcium store by the GFs was suppressed in parallel with the calcium oscillation.

\section{Morphology and calcium response}

Under certain pathological conditions, astrocytes proliferate and become morphologically hypertrophic; this is known as differentiation into reactive astrocytes, a process in which GFs and proinflammatory cytokines are thought to be involved (Rostworowski et al., 1997; Iseki et al., 2002). To investigate the relationship between differentiation and changes in the calcium response, we performed an immunocytochemical study using anti-GFAP antibody and Hoechst nuclear staining and examined astrocyte morphology and proliferation under different culture conditions. As shown in Figure 4A, cells cultured in ADM bore more fibers staining strongly for GFAP, whereas those cultured in GF-free ADM were flat and showed mesh-like GFAP staining in the perinuclear region. IL1 $\beta$ or LPS partially suppressed the effect of GFs, i.e., the fibrous morphology and mesh-like structure were 

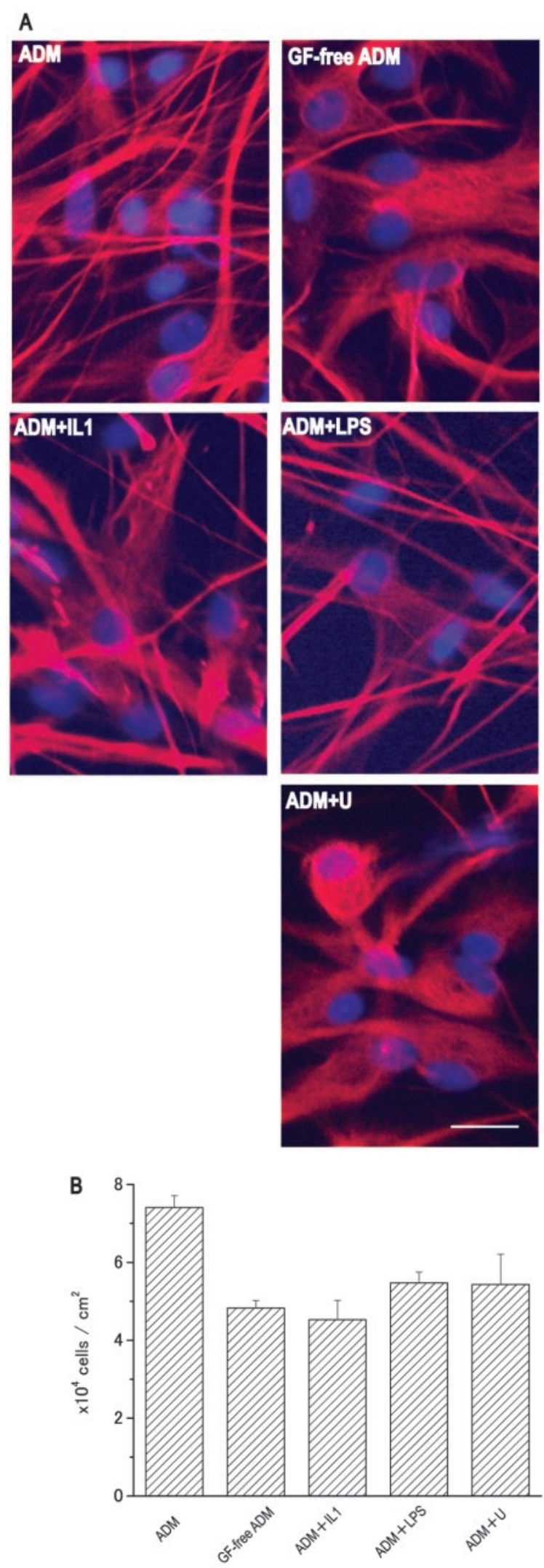

Figure 4. Morphology and proliferation of astrocytes cultured in various media. $A$, Fluorescent labeling of GFAP and nuclei, using anti-GFAP antibody and Hoechst stain. Scale bars, 20 $\mu \mathrm{m} . B$, Density of astrocytes grown in different media. The values were calculated from the number of nuclei in an area of $278 \mu \mathrm{m}^{2}$ obtained from three Hoechst-stained images and are expressed as the mean \pm SEM. The results are representative of those from three independent experiments using different series of cultures. intermediate between those in ADM and those in GF-free ADM. The effect of the MEK inhibitor was more marked, the cells being flat, as in GF-free ADM, with mesh-like GFAP fibers surrounding the nuclei. Proliferation was quantified by calculating the cell density (Fig. $4 B$ ). The GFs promoted astrocyte proliferation; the density of cells cultured in GF-free ADM was only $65 \%$ of that of cells cultured in ADM. The densities of cells cultured in ADM containing IL $\beta$, LPS, or the MEK inhibitor were 61,73 , or $73 \%$, respectively, of that of cells grown in $\mathrm{ADM}$, indicating suppression of the GF-induced proliferation by these compounds. These results show a correlation between proliferation and calcium oscillation of astrocytes. Expression of GFAP, which increases in the reactive astrocyte in situ (Brock and O'Callaghan, 1987), was measured under the different culture conditions using Western blotting, but no significant differences were detected (data not shown). These results show that the GFs promoted proliferation and a hypertrophic morphology of astrocytes via the MAPK cascade and that these effects were blocked by pro-inflammatory cytokines and LPS. These changes correlated well with calcium oscillation, suggesting that calcium oscillation is a characteristic of a differentiation state of the astrocyte, possibly a reactive astrocyte. In addition, the results shown in Figure $4 B$ support the idea that astrocytes giving either transient or oscillatory responses are both derived from a single cell population. Although it is possible that the astrocytes with different responding patterns belong to different cell populations and that the GFs affect the proliferation of each population to a different extent, our results suggest this is not the case. The cell density, which was $3 \times 10^{4}$ cells $/ \mathrm{cm}^{2}$ at seeding, increased to $4.8 \pm 0.2 \times 10^{4}$ or $7.4 \pm 0.3 \times$ $10^{4}$ cells $/ \mathrm{cm}^{2}$ in the absence or presence of GFs, respectively. Because the percentage of cells (responders and nonresponders) showing a nonoscillatory response decreased from $\sim 90$ to $30 \%$ in the presence of GFs (Fig. 2), the GFs caused a decrease in the density of nonoscillatory cells from $4.3 \times 10^{4}$ to $2.2 \times 10^{4}$ cells/ $\mathrm{cm}^{2}$. Because GFs caused no significant cell death, and GFs would not be expected to suppress the proliferation, this reduction indicates that the GFs converted cells showing a nonoscillatory response to cells showing an oscillatory response.

\section{MAPK and the immediate early gene}

To examine the involvement of the MAPK cascade in these changes in the astrocyte, its activation was examined at two different levels, ERK phosphorylation and activation of the immediate early gene (IEG), in astrocytes cultured in the presence of factors affecting calcium dynamics. As shown in Figure $5 A$, in the presence of the GFs, ERK was phosphorylated within $5 \mathrm{~min}$; this effect was slightly enhanced by the cytokines and LPS, which did not themselves activate ERK, although they have been reported to activate this cascade in astrocytes (Molina-Holgado et al., 2000), but was abolished completely by pretreatment with the MEK inhibitor. Furthermore, we monitored gene activation via the MAPK cascade in a reporter gene assay using the IEG promoter. egr-1, which has six serum response elements and two cAMP response elements in the promoter region and encodes a transcription factor that is known to control bFGF production via the MAPK cascade in astrocytes, was used to construct the reporter gene vector (Changelian et al., 1989; Biesiada et al., 1996; Harada et al., 1996). As shown in Figure 5B, the GFs caused gene activation within $6 \mathrm{hr}$, and this was suppressed by pretreatment with the pro-inflammatory cytokines, LPS, or the MEK inhibitor. As in the ERK phosphorylation experiment, the cytokines or LPS did not themselves activate the reporter gene (data not shown). These results show that the GFs activated the MAPK cascade and sub- 


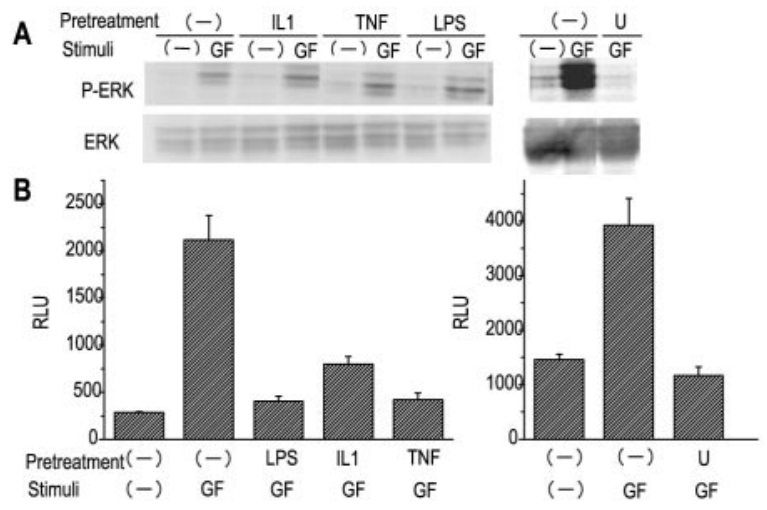

Figure 5. Activation of the MAP kinase cascade by growth factors and inhibition by proinflammatory cytokines. Astrocytes cultured in GF-free ADM $(-)$ were stimulated with growth factors (GF) for $5 \mathrm{~min}$ for Western blot experiments or for $6 \mathrm{hr}$ for reporter gene assays, with or without 5 min pretreatment with MEK inhibitor U0126 (U) or overnight pretreatment with pro-inflammatory cytokines (IL1 or TNF) or LPS. A, ERK activation monitored by Western blotting using anti-phosphorylated ERK antibody and anti-ERK antibody. $B$, Activation of the immediate early gene egr- 1 assessed by the reporter gene assay.

sequent IEG expression and that the cytokines and LPS affected these processes downstream of ERK activation and suppressed gene regulation in the cascade.

\section{Calcium oscillation in slice preparations}

To elucidate the role of the astrocytic calcium response in brain function, we investigated changes in neuronal network activity after astrocyte stimulation. In hippocampal acute slice preparations, an mGluR agonist, (1S,3R)-1-aminocyclopentane-1,3dicarboxylic acid (tACPD), induces a calcium response in astrocytes and then in neighboring neurons, and because ionotropic GluR (iGluR) antagonists inhibit only the neuronal calcium increase, it was suggested that tACPD induces both a calcium increase in, and glutamate release by, astrocytes and that these astrocyte responses affect the surrounding neurons via neuronal iGluRs (Pasti et al., 1997; Haydon, 2001). In the present study, the NMDA receptor antagonist MK801 was used to reduce the excitotoxicity of calcium indicator loading of slice preparations, in which the detergent Cremophore was used. We also noted, however, that the washing out of MK801 after loading induced spontaneous, fast, synchronous calcium increases in neurons, in accordance with a previous report that NMDA receptor blockade increases NMDA receptor expression and that the removal of the blocker induces epileptic neuronal activity (Goldin et al., 2001); therefore, we were able to use these neuronal calcium increases to investigate the effect of mGluR activation. Consequently, we used calcium imaging of individual pyramidal neurons in slice culture preparations of the neonatal rat hippocampal CA1 region to examine the effect of agents that induce an astrocyte calcium response. Figure 6 shows representative fluorescence images (Fig. $6 A)$ and time-dependent fluorescence changes in the eight pyramidal neurons marked $1-8$ in Figure $6 A e$ (Fig. $6 B-D$ ). Fluorescence images before, and at the peak of, the spontaneous, fast, synchronous calcium increase seen after MK801 washout, derived from imaging data in movie 2 (supplementary data), are shown in Figure 6, $A a$ and $A b$, respectively. The time-dependent changes in the spontaneous responses before $\mathrm{mGluR}$ activation (Fig. 6B; shown on an expanded scale in Fig. $6 C$ ) also show the synchronous spike-like properties of these calcium increases. Because the spontaneous, fast calcium increase was blocked either by a combination of two iGluR antagonists, 6-cyano-7-
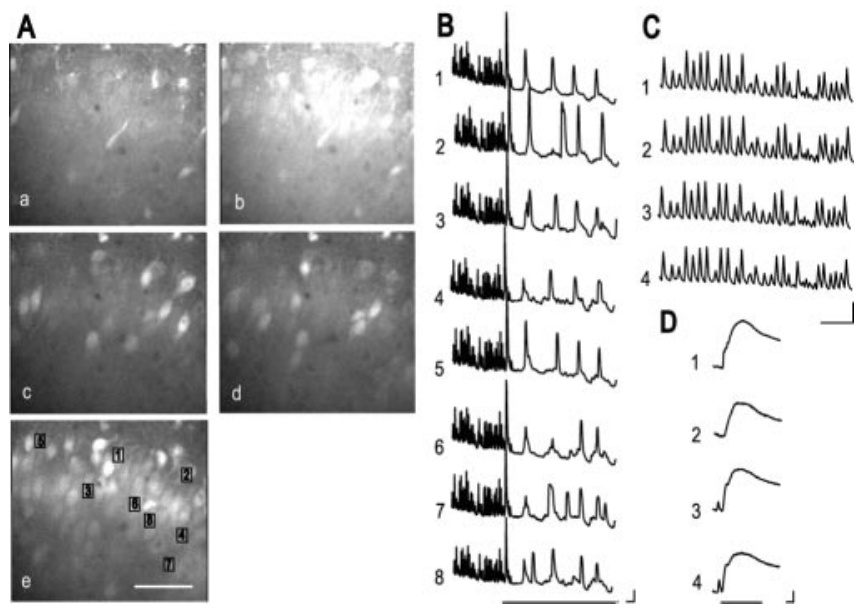

Figure 6. Generation of an oscillatory calcium response in neurons in the CA1 region of rat hippocampal slice preparations, similar to that seen in cultured astrocytes. $A$, Calcium increase in neurons monitored by confocal microscopy: spontaneous, fast, synchronous calcium increase seen on MK801 washout $(a, b)$; calcium oscillation after application of $30 \mu \mathrm{M} \operatorname{tACPD}(c, d)$; transient and plateau calcium response to $1 \mathrm{~mm}$ glutamate (e). Scale bar, $50 \mu \mathrm{m}$. $B-D$, Spontaneous and tACPD-induced calcium responses of the cells marked 1- 8 in $A$ e. $B$, Conversion of the calcium response after $t A C P D$ application, indicated by the line below the traces. $C$, The spontaneous calcium increase before $\mathrm{tACPD}$ application on an expanded time scale. $D$, The calcium increase in response to $1 \mathrm{~mm}$ glutamate. Calibration: horizontal, $10 \mathrm{sec}$; vertical $(F / F 0)$, 0.1 in $B$ and $C$ and 0.2 in $D$.

nitroquinoxaline-2,3-dione $(50 \mu \mathrm{M})$ and $\mathrm{D}$-(-)-2-amino-5phosphonopentanoic acid $(50 \mu \mathrm{M})$, or by the sodium channel blocker tetrodotoxin $(1 \mu \mathrm{M})$ (data not shown), it was probably caused by the synchronous activity of neurons as a result of synaptic transmission. Activation of mGluRs by tACPD significantly affected the calcium response, i.e., the synchronized fast calcium increase was converted to an asynchronous slow calcium oscillation (Fig. 6B). Two representative fluorescence images of the calcium oscillation derived from movie 3 (supplementary data) are shown in Figure 6, $A c$ and $A d$. These results show that tACPD treatment reduced the frequency of the calcium response and made each response independent. Subsequent application of a high concentration of glutamate $(1 \mathrm{~mm})$ induced a global calcium increase (Fig. $6 A e, D$ ), and most of the neurons could be identified. The frequency of the slow calcium increase in the neurons in the slice preparation after tACPD application $(1.71 \pm 0.11$ peaks per minute; $n=8$ cells) was similar to that seen in astrocytes cultured in ADM, as described above $(2.05 \pm 0.14$ peaks per minute; $n=228$ cells). These results suggest that the intrinsic calcium oscillation in astrocytes influences neuronal activity and supports the idea that the astrocytic calcium response to tACPD results in glutamate release, which induces a calcium response in neighboring neurons via iGluRs, as proposed on the basis of similar experiments using acute slice preparations (Pasti et al., 1997). Furthermore, the present results show that mGluR activation converts the pattern of neuronal activity and suggest that astrocytes play a dominant role in the neuronal calcium increase after mGluR activation.

\section{Discussion}

On the basis of the above findings, we propose the model shown in Figure 7, in which the astrocytic calcium response is altered by soluble factors, the production of which is affected by the CNS environment, and this change in response then affects the activity of neighboring neurons. The GFs, EGF and bFGF, increase the size of the intracellular calcium store in the astrocyte, which leads 


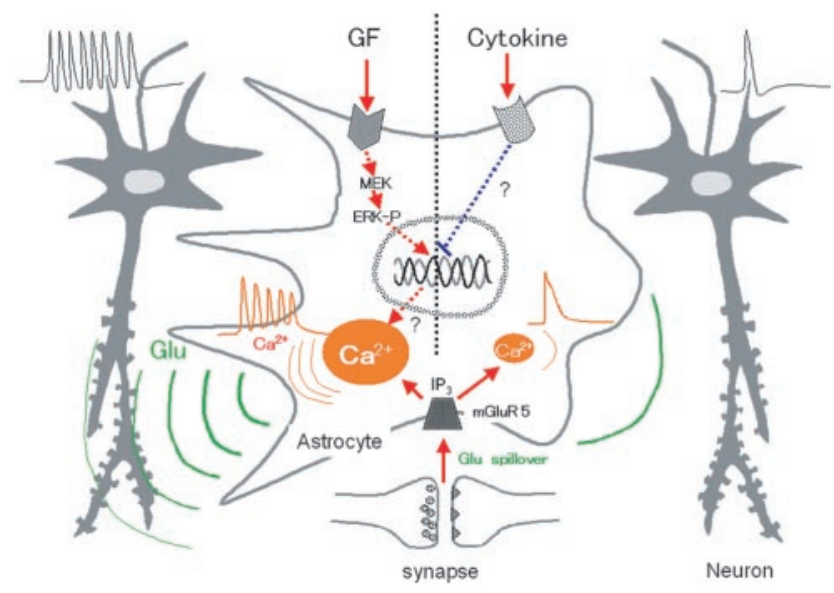

Figure 7. Proposed scheme showing the effect of astrocytes on neuronal activity. ERK-P Phosphorylated ERK.

to conversion of the neurotransmitter-induced calcium response from transient to oscillatory and promotes proliferation and a hypertrophic morphology, all of which involve the MAPK cascade. The calcium oscillation generated in the astrocyte in response to glutamate leaking from the synaptic cleft during neuronal activity causes repetitive glutamate release, which gives back repetitive excitatory inputs to the neuronal network. In contrast, pro-inflammatory cytokines suppress these changes in the calcium response and morphology by inhibiting the expression of genes downstream of the MAPK cascade. This leads to a reduction in excitatory input from astrocytes to neurons that is influenced by the frequency of glutamate release and contact between the two types of cell.

A broad spectrum of nonexcitable cells show calcium oscillation. The bell-shaped calcium dependency of the $\mathrm{IP}_{3}$ receptor (Miyakawa et al., 1999) and the dual regulation of regulator of G-protein signaling 4 by calcium and phosphatidylinositol triphosphate (Luo et al., 2001) have been suggested as the possible mechanisms, but no general model explaining all of the phenomena has been presented. Furthermore, there are reports that oscillations in intracellular $\mathrm{IP}_{3}$ levels are synchronized with calcium oscillations (Hirose et al., 1999) and that spontaneous oscillation of calcium release from the intracellular calcium store is directly stimulated by a low $\mathrm{IP}_{3}$ concentration (Hajnoczky and Thomas, 1997). The present results showed that the size of the calcium store, but not mGluR levels, was crucial in generating calcium oscillation in astrocytes. Because the GFs altered the calcium responses to both glutamate and ATP and did not affect mGluR5 expression, this shows that their effect was independent of the type and level of expression of receptors. Furthermore, the calcium response induced by direct activation of $\mathrm{IP}_{3}$ receptors by thimerosal was also converted from transient to oscillatory by the GFs, suggesting that the GFs affected the properties of the calcium store or some controlling mechanism of calcium dynamics. Measurement of the size of the calcium store using ionomycin showed that enlargement of the calcium store correlated with the generation of the oscillatory calcium response. A similar correlation has been reported in mouse oocytes during maturation (Jones et al., 1995), suggesting that this is a common mechanism for converting the response pattern under physiological conditions. We assume that GFs increase the size of the calcium store and then increase the duration or total amount of calcium release, which finally affects the local calcium concentration around the $\mathrm{IP}_{3}$ receptor. Because the $\mathrm{IP}_{3}$ receptor is regulated by calcium in both a positive and negative manner (Miyakawa et al., 1999), GFs may affect $\mathrm{IP}_{3}$ receptor function via the local calcium concentration and produce synchronized calcium release. Another possible explanation for the calcium oscillation is that when GFs increase the size and possibly the distribution of the calcium stores, this may enable the propagation of a calcium wave, which is thought to be one mechanism involved in calcium oscillation (Carafoli, 2002). If enlargement of the calcium store resulted in a larger region of the astrocyte being involved in the calcium response, it is likely that the local calcium increase propagates as a calcium wave. Some cases of calcium oscillation have been explained as a result of repetitive propagation of calcium waves (Miyazaki et al., 1992; Strahonja-Packard and Sanderson, 1999), and propagation of the calcium increase was observed during calcium oscillation (see movie 1 in supplementary data). Further analysis of the calcium store in astrocytes, including the calcium concentration in the store in both the resting and stimulated states, the morphology of the endoplasmic reticulum, and the localization of the $\mathrm{IP}_{3}$ receptor, will provide useful information for examining these two possibilities.

The above-described regulation of calcium oscillation in the astrocyte by GFs and pro-inflammatory cytokines is the first evidence for the dual regulation of calcium dynamics by soluble factors and could be the mechanism by which the astrocyte detects changes in the CNS environment and regulates brain activities, such as the processes of inflammation, regeneration, and memory formation, under various physiological conditions. Because the GFs promoted proliferation and a hypertrophic morphology, in addition to calcium oscillation, an oscillatory calcium response to neurotransmitters could be a property of reactive astrocytes. If this is the case, neurodegeneration during gliosis could be attributed to this calcium oscillation of the astrocyte, which would result in increased glutamate release and cause excitotoxicity. We cannot definitely conclude that the properties of astrocytes cultured in ADM reflect those of reactive astrocytes, however, because the GFs did not cause increased expression of GFAP, which is reported to be increased in reactive astrocytes (Brock and O'Callaghan, 1987), and it is known that both GFs and pro-inflammatory cytokines are involved in the differentiation of reactive astrocytes (Rostworowski et al., 1997; Iseki et al., 2002). GFs are produced to some extent in the CNS under normal physiological conditions and act as tropic factors, and their concentrations are altered in response to physical and psychological conditions (Stachowiak et al., 1997; Gomez-Pinilla et al., 1998; Xian and Zhou, 1999). In contrast, pro-inflammatory cytokine production is suppressed until triggered by events such as brain damage, psychological stress, or aging (Rostworowski et al., 1997; Murray and Lynch, 1998). On the basis of these two lines of evidence, the percentage of astrocytes showing an oscillatory calcium response is assumed to vary in the normal CNS, mostly depending on the production of GFs, as seen in cells cultured in the presence of $10 \%$ FCS. This flexibility in the calcium response could be part of the regulatory mechanism of memory formation, because the astrocytic calcium response to neuronal activity, especially tetanic stimulation, is reported to affect synaptic plasticity (Kang et al., 1998). This notion is in good agreement with the evidence that synaptic transmission is promoted by GFs (Ishiyama et al., 1991) but reduced by pro-inflammatory cytokines (Murray and Lynch, 1998). For both sets of factors, the astrocyte would be the main target for regulation of higher brain function. This dual regulation of the MAPK cascade was shown to be crucial in all of the processes described in the present study, and our 
results are evidence for a novel regulatory mechanism in this cascade, because the presently known negative regulation mechanisms for the MAPK cascade affect signaling between receptors and ERKs, such as MAPK phosphatases (Takaki et al., 2001) and sprouty (Hanafusa et al., 2002).

Most mGluRs have a 10-fold higher affinity than iGluRs for glutamate (Conn and Pin, 1997), thus allowing a long-lasting calcium response (including calcium oscillation) by the astrocyte to glutamate leaked from the synaptic cleft after massive neuronal activation, e.g., by tetanic stimulation (Porter and McCarthy, 1996). Furthermore, because neuronal mGluRs are localized in the perisynaptic region (Baude et al., 1993; Lujan et al., 1997), they are probably activated by the leaked glutamate (Lujan et al., 1997; Mitchell and Silver, 2000). In the present study on slice cultures, the mGluR agonist tACPD converted the spontaneous, fast, synchronous calcium increase of pyramidal neurons to slow, asynchronous calcium oscillation. Furthermore, the frequency of the calcium oscillation in the neurons in the slice cultures was similar to that of cultured astrocytes, suggesting that the intrinsic calcium oscillation in astrocytes influences neighboring neurons via glutamate release. These results imply that the astrocyte plays a dominant role in neuronal activity after $m$ GluR activation. Further examination of the effects of soluble factors on calcium responses in slice cultures using serum-free medium should reveal how they affect neuronal activity via changes in the astrocyte. Although calcium-dependent glutamate release from astrocytes has been shown in both culture and slice preparation (Bezzi et al., 2001), it is reported to have both excitatory and inhibitory effects on neuronal activity (Porter and McCarthy, 1996; Araque et al., 1998), and its real consequence still has to be determined. It is reasonable to assume that the response of neurons to glutamate released by astrocytes is dependent on the subtype of glutamate receptor, which can vary outside synapses, and that the inhibitory effects are caused by inhibitory mGluRs (groups II and III). If this were the case, the physiological role of the calcium response and glutamate release by the astrocyte would vary, depending on the structure and topology of the glutamate release site on the astrocyte and the glutamate-receptive site on the neuron. In conclusion, we propose that the soluble factor-mediated regulation of astrocyte calcium dynamics is a novel mechanism for sensing the state of the CNS environment and responding to it by altering the physiology and pathology of the CNS. Additional studies on this regulatory mechanism should provide significant information on how the brain works.

\section{References}

Araque A, Parpura V, Sanzgiri RP, Haydon PG (1998) Glutamatedependent astrocyte modulation of synaptic transmission between cultured hippocampal neurons. Eur J Neurosci 10:2129-2142.

Baude A, Nusser Z, Roberts JD, Mulvihill E, McIlhinney RA, Somogyi P (1993) The metabotropic glutamate receptor (mGluR1 alpha) is concentrated at perisynaptic membrane of neuronal subpopulations as detected by immunogold reaction. Neuron 11:771-787.

Bezzi P, Domercq M, Brambilla L, Galli R, Schols D, De Clercq E, Vescovi A, Bagetta G, Kollias G, Meldolesi J, Volterra A (2001) CXCR4-activated astrocyte glutamate release via TNFalpha: amplification by microglia triggers neurotoxicity. Nat Neurosci 4:702-710.

Biesiada E, Razandi M, Levin ER (1996) Egr-1 activates basic fibroblast growth factor transcription. Mechanistic implications for astrocyte proliferation. J Biol Chem 271:18576-18581.

Brock TO, O'Callaghan JP (1987) Quantitative changes in the synaptic vesicle proteins synapsin I and p38 and the astrocyte-specific protein glial fibrillary acidic protein are associated with chemical-induced injury to the rat central nervous system. J Neurosci 7:931-942.

Cai Z, Schools GP, Kimelberg HK (2000) Metabotropic glutamate receptors in acutely isolated hippocampal astrocytes: developmental changes of mGluR5 mRNA and functional expression. Glia 29:70-80.

Carafoli E (2002) Calcium signaling: a tale for all seasons. Proc Natl Acad Sci USA 99:1115-1122.

Changelian PS, Feng P, King TC, Milbrandt J (1989) Structure of the NGFI-A gene and detection of upstream sequences responsible for its transcriptional induction by nerve growth factor. Proc Natl Acad Sci USA 86:377-381.

Conn PJ, Pin JP (1997) Pharmacology and functions of metabotropic glutamate receptors. Annu Rev Pharmacol Toxicol 37:205-237.

Favata MF, Horiuchi KY, Manos EJ, Daulerio AJ, Stradley DA, Feeser WS, Van Dyk DE, Pitts WJ, Earl RA, Hobbs F, Copeland RA, Magolda RL, Scherle PA, Trzaskos JM (1998) Identification of a novel inhibitor of mitogen-activated protein kinase kinase. J Biol Chem 273:18623-18632.

Goldin M, Segal M, Avignone E (2001) Functional plasticity triggers formation and pruning of dendritic spines in cultured hippocampal networks. J Neurosci 21:186-193.

Gomez-Pinilla F, So V, Kesslak JP (1998) Spatial learning and physical activity contribute to the induction of fibroblast growth factor: neural substrates for increased cognition associated with exercise. Neuroscience 85:53-61.

Hajnoczky G, Thomas AP (1997) Minimal requirements for calcium oscillations driven by the IP3 receptor. EMBO J 16:3533-3543.

Hanafusa H, Torii S, Yasunaga T, Nishida E (2002) Sprouty1 and Sprouty2 provide a control mechanism for the Ras/MAPK signalling pathway. Nat Cell Biol 4:850-858.

Harada S, Smith RM, Smith JA, White MF, Jarett L (1996) Insulin-induced egr-1 and c-fos expression in 32D cells requires insulin receptor, Shc, and mitogen-activated protein kinase, but not insulin receptor substrate-1 and phosphatidylinositol 3-kinase activation. J Biol Chem 271:30222-30226.

Haydon PG (2001) GLIA: listening and talking to the synapse. Nat Rev Neurosci 2:185-193.

Herx LM, Yong VW (2001) Interleukin-1 beta is required for the early evolution of reactive astrogliosis following CNS lesion. J Neuropathol Exp Neurol 60:961-971.

Hinkle DA, Harney JP, Cai A, Hilt DC, Yarowsky PJ, Wise PM (1998) Basic fibroblast growth factor-2 and interleukin-1 beta regulate S100 beta expression in cultured astrocytes. Neuroscience 82:33-41.

Hirasawa T, Nakamura T, Mizushima A, Morita M, Ezawa I, Miyakawa H, Kudo Y (2000) Adverse effects of an active fragment of parathyroid hormone on rat hippocampal organotypic cultures. $\mathrm{Br} \mathrm{J}$ Pharmacol 129:21-28.

Hirose K, Kadowaki S, Tanabe M, Takeshima H, Iino M (1999) Spatiotemporal dynamics of inositol 1,4,5-trisphosphate that underlies complex $\mathrm{Ca}^{2+}$ mobilization patterns. Science 284:1527-1530.

Iseki K, Hagino S, Mori T, Zhang Y, Yokoya S, Takaki H, Tase C, Murakawa M, Wanaka A (2002) Increased syndecan expression by pleiotrophin and FGF receptor-expressing astrocytes in injured brain tissue. Glia 39:1-9.

Ishiyama J, Saito H, Abe K (1991) Epidermal growth factor and basic fibroblast growth factor promote the generation of long-term potentiation in the dentate gyrus of anaesthetized rats. Neurosci Res 12:403-411.

Jensen AM, Chiu SY (1990) Fluorescence measurement of changes in intracellular calcium induced by excitatory amino acids in cultured cortical astrocytes. J Neurosci 10:1165-1175.

Jones KT, Carroll J, Whittingham DG (1995) Ionomycin, thapsigargin, ryanodine, and sperm induced $\mathrm{Ca}^{2+}$ release increase during meiotic maturation of mouse oocytes. J Biol Chem 270:6671-6677.

Kang J, Jiang L, Goldman SA, Nedergaard M (1998) Astrocyte-mediated potentiation of inhibitory synaptic transmission. Nat Neurosci 1:683-692.

Krushel LA, Tai MH, Cunningham BA, Edelman GM, Crossin KL (1998) Neural cell adhesion molecule (N-CAM) domains and intracellular signaling pathways involved in the inhibition of astrocyte proliferation. Proc Natl Acad Sci USA 95:2592-2596.

Levison SW, McCarthy KD (1991) Astroglia in culture. In: Culturing nerve cells (Banker G, Goslin K, eds), pp 309-336. Cambridge, MA: MIT.

Lujan R, Roberts JD, Shigemoto R, Ohishi H, Somogyi P (1997) Differential plasma membrane distribution of metabotropic glutamate receptors mGluR1 alpha, mGluR2 and mGluR5, relative to neurotransmitter release sites. J Chem Neuroanat 13:219-241.

Luo X, Popov S, Bera AK, Wilkie TM, Muallem S (2001) RGS proteins pro- 
vide biochemical control of agonist-evoked $[\mathrm{Ca} 2+] \mathrm{i}$ oscillations. Mol Cell 7:651-660.

McCarthy KD, Salm AK (1991) Pharmacologically-distinct subsets of astroglia can be identified by their calcium response to neuroligands. Neuroscience 41:325-333.

Miller S, Romano C, Cotman CW (1995) Growth factor upregulation of a phosphoinositide-coupled metabotropic glutamate receptor in cortical astrocytes. J Neurosci 15:6103-6109.

Mitchell SJ, Silver RA (2000) Glutamate spillover suppresses inhibition by activating presynaptic mGluRs. Nature 404:498-502.

Miyakawa T, Maeda A, Yamazawa T, Hirose K, Kurosaki T, Iino M (1999) Encoding of $\mathrm{Ca}^{2+}$ signals by differential expression of IP3 receptor subtypes. EMBO J 18:1303-1308.

Miyazaki S, Yuzaki M, Nakada K, Shirakawa H, Nakanishi S, Nakade S, Mikoshiba K (1992) Block of $\mathrm{Ca}^{2+}$ wave and $\mathrm{Ca}^{2+}$ oscillation by antibody to the inositol 1,4,5-trisphosphate receptor in fertilized hamster eggs. Science 257:251-255.

Molina-Holgado E, Ortiz S, Molina-Holgado F, Guaza C (2000) Induction of COX-2 and PGE(2) biosynthesis by IL-1beta is mediated by PKC and mitogen-activated protein kinases in murine astrocytes. $\mathrm{Br} \mathrm{J}$ Pharmacol 131:152-159.

Morita M, Susuki J, Moto T, Higuchi C, Kudo Y (2003) A novel method to quantify calcium response pattern and oscillation using Fura2 and acridine orange. J Pharmacol Sci, in press.

Muller W, Heinemann U, Berlin K (1997) Cholecystokinin activates CCKBreceptor-mediated Ca-signaling in hippocampal astrocytes. J Neurophysiol 78:1997-2001.

Murray CA, Lynch MA (1998) Evidence that increased hippocampal expression of the cytokine interleukin-1 beta is a common trigger for ageand stress-induced impairments in long-term potentiation. J Neurosci 18:2974-2981.

Nakahara K, Okada M, Nakanishi S (1997) The metabotropic glutamate receptor mGluR5 induces calcium oscillations in cultured astrocytes via protein kinase C phosphorylation. J Neurochem 69:1467-1475.

Pasti L, Volterra A, Pozzan T, Carmignoto G (1997) Intracellular calcium oscillations in astrocytes: a highly plastic, bidirectional form of communication between neurons and astrocytes in situ. J Neurosci 17:7817-7830.

Pasti L, Zonta M, Pozzan T, Vicini S, Carmignoto G (2001) Cytosolic calcium oscillations in astrocytes may regulate exocytotic release of glutamate. J Neurosci 21:477-484.

Porter JT, McCarthy KD (1996) Hippocampal astrocytes in situ respond to glutamate released from synaptic terminals. J Neurosci 16:5073-5081.

Raetz CR, Whitfield C (2002) Lipopolysaccharide endotoxins. Annu Rev Biochem 71:635-700.

Rostworowski M, Balasingam V, Chabot S, Owens T, Yong VW (1997) Astrogliosis in the neonatal and adult murine brain post-trauma: elevation of inflammatory cytokines and the lack of requirement for endogenous interferon- $\gamma$. J Neurosci 17:3664-3674.

Stachowiak MK, Moffett J, Maher P, Tucholski J, Stachowiak EK (1997) Growth factor regulation of cell growth and proliferation in the nervous system. A new intracrine nuclear mechanism. Mol Neurobiol 15:257-283.

Strahonja-Packard A, Sanderson MJ (1999) Intercellular $\mathrm{Ca}(2+)$ waves induce temporally and spatially distinct intracellular $\mathrm{Ca}(2+)$ oscillations in glia. Glia 28:97-113.

Swann K (1991) Thimerosal causes calcium oscillations and sensitizes calcium-induced calcium release in unfertilized hamster eggs. FEBS Lett 278:175-178.

Takaki M, Ujike H, Kodama M, Takehisa Y, Nakata K, Kuroda S (2001) Two kinds of mitogen-activated protein kinase phosphatases, MKP-1 and MKP-3, are differentially activated by acute and chronic methamphetamine treatment in the rat brain. J Neurochem 79:679-688.

Uchida Y (1999) Regulation of growth inhibitory factor expression by epidermal growth factor and interleukin-1beta in cultured rat astrocytes. J Neurochem 73:1945-1953.

Verkhratsky A, Orkand RK, Kettenmann H (1998) Glial calcium: homeostasis and signaling function. Physiol Rev 78:99-141.

Xian CJ, Zhou XF (1999) Roles of transforming growth factor-alpha and related molecules in the nervous system. Mol Neurobiol 20:157-183.

Zonta M, Angulo MC, Gobbo S, Rosengarten B, Hossmann KA, Pozzan T, Carmignoto G (2003) Neuron-to-astrocyte signaling is central to the dynamic control of brain microcirculation. Nat Neurosci 6:43-50. 